Imperial/TP/94-95/22

gr-qc/9502036

\title{
Completeness of Decoherence Functionals
}

\author{
Stephan Schreckenberg[ \\ Blackett Laboratory \\ Imperial College \\ South Kensington \\ London SW7 2BZ
}

February 1995

\begin{abstract}
The basic ingredients of the 'consistent histories' approach to a generalized quantum theory are 'histories' and decoherence functionals. The main aim of this program is to find and to study the behaviour of consistent sets associated with a particular decoherence functional $d$. In its recent formulation by Isham [四] it is natural to identify the space $\mathcal{U P}$ of propositions about histories with an orthoalgebra or lattice. When $\mathcal{U P}$ is given by the lattice of projectors $\mathcal{P}(\mathcal{V})$ in some Hilbert space $\mathcal{V}$, consistent sets correspond to certain partitions of the unit operator in $\mathcal{V}$ into mutually orthogonal projectors $\left\{\alpha_{1}, \alpha_{2}, \ldots\right\}$, such that the function $d(\alpha, \alpha)$ is a probability distribution on the boolean algebra generated by $\left\{\alpha_{1}, \alpha_{2}, \ldots\right\}$. Using the classification theorem for decoherence functionals proven in [6] we show that in the case where $\mathcal{V}$ is some separable Hilbert space there exists for each partition of the unit operator into a set of mutually orthogonal projectors, and for any probability distribution $p(\alpha)$ on the corresponding boolean algebra, decoherence functionals $d$ with respect to which this set is consistent and which are such that for the probability functions $d(\alpha, \alpha)=p(\alpha)$ holds.
\end{abstract}

PACS numbers: 03.65.Bz, 03.65.Ca, 03.65.Db

\footnotetext{
${ }^{1}$ email: stschr@ic.ac.uk
} 


\section{INTRODUCTION}

Over the past three years the 'consistent histories' approach to quantum theory, initiated by the work of Griffiths [可, Omnès [2], Gell-Mann and Hartle [3], has been subject of intense research. The motivations for these activities are diverse and include hopes that the new conceptual framework advocated will be general enough to resolve issues connected with the measurement problem and the quantum theory of closed systems, as well as giving new insights into the problem of constructing a quantum theory of gravity.

The two basic ingredients in this scheme are a 'space of histories' $\mathcal{U P}$ and a 'space of decoherence functionals' $\mathcal{D}$, whose elements are complex-valued functions on pairs of histories.

Recall how these spaces arise in non-relativistic quantum mechanics. Let us suppose we are given a Hilbert space $\mathcal{H}$, a Hamiltonian operator $H$ and a density matrix $\rho_{t_{0}}$ decribing the state at some initial time $t_{0}$. Then the joint probability of finding all the properties $\alpha:=\left(\alpha_{t_{1}}, \alpha_{t_{2}}, \ldots, \alpha_{t_{n}}\right)$ with $t_{1}<t_{2}<\cdots<t_{n}$, given by the Schrödinger picture projection operators $\left\{\alpha_{t_{i}}\right\}$, in a time-ordered sequence of measurements is given by:

$$
d_{(H, \rho)}(\alpha, \alpha)=\operatorname{tr}_{\mathcal{H}}\left(\tilde{C}_{\alpha}^{\dagger} \rho_{t_{0}} \tilde{C}_{\alpha}\right)
$$

where the 'class' operator $\tilde{C}_{\alpha}$ is defined to be

$$
\tilde{C}_{\alpha}:=\alpha_{t_{1}}\left(t_{1}\right) \alpha_{t_{2}}\left(t_{2}\right) \cdots \alpha_{t_{n}}\left(t_{n}\right)
$$

with $\left\{\alpha_{t_{i}}\left(t_{i}\right):=e^{\frac{i}{\hbar} H\left(t_{i}-t_{0}\right)} \alpha_{t_{i}} e^{-\frac{i}{\hbar} H\left(t_{i}-t_{0}\right)}\right\}$ being the associated Heisenberg picture operators. This set $\alpha:=\left(\alpha_{t_{1}}, \alpha_{t_{2}}, \ldots, \alpha_{t_{n}}\right)$ is called a homogeneous history.

In the consistent histories approach one assumes that the assignment of probabilities to histories is still meaningful but only for certain subsets of the space of histories. These subsets are selected through the requirement that the central object, a complex-valued decoherence functional $d(\alpha, \beta) \in \mathcal{D}$, like for example

$$
d_{(H, \rho)}(\alpha, \beta)=\operatorname{tr}_{\mathcal{H}}\left(\tilde{C}_{\alpha}^{\dagger} \rho_{t_{0}} \tilde{C}_{\beta}\right),
$$

satisfies the so called consistency conditions when evaluated on pairs $(\alpha, \beta)$ of elements of those subsets. The virtue is that one can talk about the elements of consistent sets as posessing definite properties. Notice that, as suggested by the notation, the initial state, $\rho_{t_{0}}$, and the dynamical structure, i.e. $H$, are coded into the decoherence functional $d \in \mathcal{D}$. This analysis in terms of a 'passive' set of histories and an 'active' set of decoherence functionals allows us to make precise statements about both spaces.

In order to give a proper meaning to the expressions just introduced, as well as to the heuristic operations of taking disjoint sums, negation and coarse-graining on the space of histories for the general theory, as they had been introduced by Gell-Mann and Hartle, it was first suggested by Isham in 1 that the set of histories $\mathcal{U P}$, more precisely the set of 
propositions about 'histories' or 'universes', can be described by an orthoalgebra.

The argument for proposing this structure is that the minimal mathematical object on which such relations can be defined seems to be an orthoalgebra whose algebraic operations $(\oplus, \neg, \leq)$ correspond to the aforementioned operations and which has elements $\mathbf{0}, \mathbf{1} \in \mathcal{U P}$ such that $\mathbf{0} \leq \alpha \leq \mathbf{1}$ for all $\alpha \in \mathcal{U P}$.

Note that the word 'history' or 'universe' is just a label for elements of $\mathcal{U P}$; it does not necessarily imply any temporal properties.

An example where such an algebra arises is provided by standard quantum mechanics when formulated as a history theory. In this case the set of all history-propositions $\mathcal{U P}$ can indeed be identified with the lattice of projectors on a certain Hilbert space (四, 可] and section IV).

In terms of the algebraic structure on the space $\mathcal{U P}$ the defining properties for decoherence functionals are as follows.

Any decoherence functional $d: \mathcal{U P} \times \mathcal{U P} \rightarrow \mathbb{C}$ has to satisfy the following conditions:

1. Hermiticity: $d(\alpha, \beta)=d(\beta, \alpha)^{*}$ for all $\alpha, \beta$.

2. Positivity: $d(\alpha, \alpha) \geq 0$ for all $\alpha$.

3. Additivity: $d(\alpha \oplus \beta, \gamma)=d(\alpha, \gamma)+d(\beta, \gamma)$ for all $\gamma$, whenever $\alpha$ and $\beta$ are disjoint. If appropriate, this can be extended to countable sums.

4. Normalisation: $d(1,1)=1$.

We emphazise that from these requirements alone it does not necessarily follow that in the case of standard quantum mechanics these functionals must be of the form (11.3), even if one adds a final density matrix or replaces the unitary evolution by a non-unitary one $[5$.

In the 'consistent histories' programme one is particularly interested in boolean subalgebras $\mathcal{A}$ of $\mathcal{U P}$, which contain the unit element and which are associated with a particular decoherence functional $d$ in such a way that the function $d(\alpha, \alpha)$ is a probability distribution on $\mathcal{A}$. To find such algebras one imposes 'consistency conditions' on the values of the decoherence functional $d$ on pairs of history propositions which can serve as generators of boolean subalgebras of $\mathcal{U P}$. If an algebra $\mathcal{A}$ is found by this procedure its generators are often referred to as a 'consistent set of history propositions with respect to $d$ '.

Therefore, a consistent set obtained in such a way carries two pieces of information:

1. It is a consistent set; it has been obtained by fulfilling the consistency conditions associated with a particular decoherence functional $d \in \mathcal{D}$.

2. It carries a probability function $p_{d}(\alpha):=d(\alpha, \alpha)$.

The aim of the present paper is to investigate whether or not this information in turn is sufficient to determine the decoherence functional $d$ from which this set arose. More specifically, we ask the following questions: 
- For which boolean subalgebra $\mathcal{A}$ of $\mathcal{U P}$ and which probability distributions $p(\alpha)$ on $\mathcal{A}$ does there exist a decoherence functional $d \in \mathcal{D}$ such that this pair can be obtained from a consistent set with respect to $d$ ?

- If such a decoherence functional $d$ exists, is it uniquely associated with the pair $(\mathcal{A}, p(\alpha))$ ?

Given any separable Hilbert space $\mathcal{V}$, its lattice of projection operators $\mathcal{P}(\mathcal{V})$ forms an orthoalgebra and can thus serve as an example for the space $\mathcal{U P}$. It is therefore of considerable interest to answer these questions for this particular model of the proposed framework.

It turns out that in this case for any such pair of a boolean algebra and a probability distribution there exist in fact many decoherence functionals which serve the purpose. This result arises as a corollary of the classification theorem for decoherence functionals proven in [6]. The value of this rather technical statement stems from its implications for the formalism to be developed.

The paper is organized as follows. In section II we provide some basic definitions and results to prepare the main result which will be proven in section III. In section IV we discuss some of its implications and finish this paper with conclusions drawn in section V.

\section{DEFINITIONS AND BASIC FACTS}

We are now going to define the notion of a consistent set of history propositions.

Definition: We call a set of history propositions $I_{n}:=\left\{\alpha_{1}, \alpha_{2}, \ldots, \alpha_{n}\right\}$ a partition of unity, if all the $\left\{\alpha_{i}\right\}$ are mutually orthogonal and add up to the unit in $\mathcal{U P}$, that is if $\mathbf{1}=\alpha_{1} \oplus \alpha_{2} \oplus \cdots \oplus \alpha_{n}$.

A set of history propositions that has the property of being a partition of unity is often referred to as being exclusive, that is its elements are mutually orthogonal, and exhaustive, that is $\oplus_{i=1}^{n} \alpha_{i}=\mathbf{1}$.

In case when $\mathcal{U P}$ is the lattice of projectors of an infinite-dimensional Hilbert space the equality has to be substituted by the requirement of weak convergence of a disjoint union of a finite or countable number of projectors to the unit operator.

Definition: Fix a decoherence functional $d \in \mathcal{D}$. If there exists a partition of unity $I_{n}$ in $\mathcal{U P}$, such that for all $\alpha_{i} \in I_{n}$ the consistency conditions given below hold, $I_{n}$ is called a consistent set of history propositions with respect to the decoherence functional $d \in \mathcal{D}$.

The conditions are:

$$
d\left(\alpha_{i}, \alpha_{j}\right)=0 \quad \text { for all } \quad i \neq j ; i, j \in\{1,2, \ldots, n\} .
$$


If there exists for a decoherence functional $d \in \mathcal{D}$ a consistent set $I_{n}$, then the properties of the decoherence functional ensure the existence of a probability distribution $p_{d}(\alpha):=d(\alpha, \alpha)$, for all history propositions $\alpha$ that belong to the boolean algebra $\mathcal{A}_{I_{n}}$ generated by the elements $\left\{\alpha_{i}\right\}$ of $I_{n}$.

The choice of consistency conditions adopted here is often referred to as 'medium decoherence'. Weaker conditions require, for example, only the real part of $d\left(\alpha_{i}, \alpha_{j}\right), i \neq j$, to vanish; $p_{d}(\alpha)$ continues to define a probability function [7]. Imposing a weaker consistency condition implies that the number of consistent sets for a $d \in \mathcal{D}$ can be larger than the number determined by conditions (2.1). In choosing the most restrictive condition we ensure the validity of our main result for all situations.

We concentrate now on the situation where the space $\mathcal{U P}$ is given by the lattice of projection operators $\mathcal{P}(\mathcal{V})$ on some finite-dimensional Hilbert space $\mathcal{V}$. In this case one can prove the following Classification Theorem for bounded decoherence functionals:

Theorem[6] If $\operatorname{dim} \mathcal{V}>2$, decoherence functionals $d$ are in one-to-one correspondence with operators $X$ on $\mathcal{V} \otimes \mathcal{V}$ according to the rule

$$
d(\alpha, \beta)=\operatorname{tr}_{\mathcal{V} \otimes \mathcal{V}}(\alpha \otimes \beta X)
$$

with the restrictions that:

$$
\begin{aligned}
& \text { a) } \operatorname{tr}_{\mathcal{V} \otimes \mathcal{V}}(\alpha \otimes \beta X)=\operatorname{tr}_{\mathcal{V} \otimes \mathcal{V}}\left(\beta \otimes \alpha X^{\dagger}\right) \text { for all } \alpha, \beta \in \mathcal{P}(\mathcal{V}), \\
& \text { b) } \operatorname{tr}_{\mathcal{V} \otimes \mathcal{V}}(\alpha \otimes \alpha X) \geq 0 \text { for all } \alpha \in \mathcal{P}(\mathcal{V}), \\
& \text { c) } \operatorname{tr}_{\mathcal{V} \otimes \mathcal{V}}(X)=1 .
\end{aligned}
$$

This is a non-trivial result since its proof requires Gleason's Theorem at various stages. Therefore conclusions drawn from it are themselves non-trivial.

\section{THE MAIN RESULT}

\section{A. The general case}

Our main result will be valid for any separable Hilbert space despite the fact that the classification theorem has not yet been extended to the infinite-dimensional case. Although it is not clear yet that in this case every decoherence functional $d \in \mathcal{D}$ is given by an operator $X$ on $\mathcal{V} \otimes \mathcal{V}$ satisfying the three conditions (2.3 - 2.5), it is nonetheless true that every operator $X$ fulfilling these conditions defines a decoherence functional; and this is all that is needed for the theorem.

\section{Theorem}


Let $\mathcal{V}$ be a separable Hilbert space and let $\mathcal{U P}$ be given by its lattice of projection operators $\mathcal{P}(\mathcal{V})$. Denote by $I=\left\{\alpha_{1}, \alpha_{2}, \ldots, \alpha_{n}\right\}$ the set of projection operators of an arbitrary partition of unity and by $p(\alpha)$ any probability distribution on the boolean algebra $\mathcal{A}_{I}$ generated by this partition. Then there exist bounded decoherence functionals $d_{I} \in \mathcal{D}$ such that $I$ is a consistent set with respect to $d_{I}$ and which are such that $d_{I}(\alpha, \alpha)=p(\alpha)$ for all $\alpha \in \mathcal{A}_{I}$.

Operators $X$ on $\mathcal{V} \otimes \mathcal{V}$ defining such $d_{I}$ are given by:

$$
X=K+\sum_{i=1}^{n} p\left(\alpha_{i}\right) \alpha_{i} \otimes \alpha_{i}
$$

with the following restrictions on the operator $K$ :

$$
\begin{array}{lll}
\text { a) } & \operatorname{tr}(\alpha \otimes \beta K)=\operatorname{tr}\left(\beta \otimes \alpha K^{\dagger}\right) & \forall \alpha, \beta \in \mathcal{P}(\mathcal{V}), \\
\text { b) } & \operatorname{tr}(\beta \otimes \beta K)+\sum_{i=1}^{n} p\left(\alpha_{i}\right)\left[\operatorname{tr}\left(\beta \alpha_{i}\right)\right]^{2} \geq 0 & \forall \beta \notin\left\{\alpha_{i}\right\}_{i=1}^{n}, \\
\text { c) } \operatorname{tr}\left(\alpha_{i} \otimes \alpha_{j} K\right)=0, \quad \operatorname{tr} K=0 & \forall i, j \in\{1,2, \ldots, n\} .
\end{array}
$$

\section{Proof}

The proof consists of checking that these operators $X$ have the required properties. Hence one has to show that conditions (2.3 - 2.5) are fulfilled to define a decoherence functional. To prove the consistency conditions (2.1), one calculates that $d_{X}\left(\alpha_{i}, \alpha_{j}\right)=0$. Finally, a short calculation shows that $d_{X}\left(\alpha_{i}, \alpha_{i}\right)=p\left(\alpha_{i}\right)$, which confirms the statement for the equality of the probability functions.

The requirements on $K$ can be met trivially by the zero-operator; but there exist other solutions. To see that it is not difficult to write those operators out it is instructive to consider the finite-dimensional case. This also fixes the notation for the discussion.

\section{B. The finite-dimensional case}

Let $\mathcal{V}$ be a Hilbert space of dimension $N<\infty$ and let $\left\{\left|e_{i}\right\rangle\right\}_{i=1}^{N}$ be one of its orthonormal bases. Then a vector-space basis for the operators on $\mathcal{V}$ is given by $\left\{B_{i j}:=\left|e_{i}\right\rangle\left\langle e_{j}\right|\right\}_{i, j=1}^{N}$, so that every operator $A$ on the tensor product space $\mathcal{V} \otimes \mathcal{V}$ can be expanded as $A=$ $\sum_{i, j, k, l=1}^{N} \lambda_{i j, k l} B_{i j} \otimes B_{k l}$ where $\lambda_{i j, k l} \in \mathbb{C}$.

\section{Lemma}

Let $\operatorname{dim} \mathcal{V}<\infty$ and let $I_{N}:=\left\{\alpha_{1}, \alpha_{2}, \ldots, \alpha_{N}\right\}$ be a partition into one-dimensional projectors; denote by $p(\alpha)$ any probability distribution on the boolean algebra $\mathcal{A}_{I_{N}}$ generated by the elements of $I_{N}$. Then there exist decoherence functionals $d_{I_{N}} \in \mathcal{D}$ such that $I_{N}$ is a consistent set with respect to these functionals $d_{I_{N}} \in \mathcal{D}$ and which are such that for the probability distribution $d_{I_{N}}(\alpha, \alpha)=p(\alpha), \forall \alpha \in \mathcal{A}_{I_{N}}$, holds. 
Without loss of generality choose the set $I_{N}$ to be given by $I_{N}:=\left\{B_{11}, B_{22}, \ldots, B_{N N}\right\}$. Then the $d_{I_{N}}$ are given by operators $X$ on $\mathcal{V} \otimes \mathcal{V}$ of the following form:

$$
X=K+\sum_{i=1}^{N} p\left(B_{i i}\right) B_{i i} \otimes B_{i i}
$$

with the restrictions that:

$$
\begin{aligned}
& \text { a) } K=\sum_{i, j=1}^{N} \kappa_{i j} B_{i j} \otimes B_{i j}, \quad \kappa_{i j} \in \mathbb{R}, \\
& \text { b) } K^{T}=K, \quad \kappa_{i i}=0, \quad \kappa_{i j} \geq 0 \quad \forall i, j \in\{1,2, \ldots, N\} .
\end{aligned}
$$

\section{Proof}

It is sufficient to consider only the case when $I_{N}$ is given by $I_{N}:=\left\{B_{11}, B_{22}, \ldots, B_{N N}\right\}$ because for any other partition of unity into one-dimensional projectors $\widetilde{I_{N}}=\left\{\widetilde{\alpha_{i}}\right\}_{i=1}^{N}$ there exists a unitary transformation $U$ on $\mathcal{V}$ relating both sets via $U B_{i i} U^{\dagger}=\widetilde{\alpha_{i}}$, for all $i \in\{1,2, \ldots, N\}$.

The corresponding $d_{\widetilde{I_{N}}}$ are given by the operators $\left[U \otimes U X U^{\dagger} \otimes U^{\dagger}\right]$ on $\mathcal{V} \otimes \mathcal{V}$.

In order to show that these $X$ define decoherence functionals with the required properties, we have to check that $K$ satisfies the conditions $3.2-3.4)$.

1. $\operatorname{tr}_{\mathcal{V} \otimes \mathcal{V}}(\alpha \otimes \beta K)=\operatorname{tr}_{\mathcal{V} \otimes \mathcal{V}}\left(\beta \otimes \alpha K^{\dagger}\right)$ for all $\alpha, \beta \in \mathcal{P}(\mathcal{V})$.

The condition $K^{T}=K$ ensures the symmetry of $K$.

Let $\alpha=\sum_{i, j=1}^{N} a_{i j} B_{i j}$ and $\beta=\sum_{i, j=1}^{N} b_{i j} B_{i j}$ be two arbitrary projectors on $\mathcal{V}$. Using the expression (3.5) for $X$ one calculates that

$$
\operatorname{tr}_{\mathcal{V} \otimes \mathcal{V}}(\alpha \otimes \beta K)=\sum_{i, j=1}^{N} a_{j i} b_{j i} \kappa_{i j}=\operatorname{tr}_{\mathcal{V} \otimes \mathcal{V}}(\beta \otimes \alpha K)
$$

2. $\operatorname{tr}(\beta \otimes \beta K)+\sum_{i=1}^{n} p\left(\alpha_{i}\right)\left[\operatorname{tr}\left(\beta \alpha_{i}\right)\right]^{2} \geq 0$ for all $\beta \notin\left\{\alpha_{i}\right\}_{i=1}^{n}$.

Equation (3.8) shows that $\operatorname{tr}_{\mathcal{V} \otimes \mathcal{V}}(\beta \otimes \beta K)=\sum_{i, j=1}^{N} b_{j i} b_{j i} \kappa_{i j}$. Since $\beta$ is a projection operator all its expansion coefficients are real. Therefore $b_{j i} b_{j i} \geq 0, \forall i, j \in$ $\{1,2, \ldots, N\}$. The condition $\kappa_{i j} \geq 0$ on the expansion coefficients of $K$ ensures that $\operatorname{tr}_{\mathcal{V} \otimes \mathcal{V}}(\beta \otimes \beta K) \geq 0$ for all $\beta \in \mathcal{P}(\mathcal{V})$, which is sufficient to fulfill the requirement.

3. $\operatorname{tr}_{\mathcal{V} \otimes \mathcal{V}}(K)=0$.

This is trivially satisfied because of $\kappa_{i i}=0$.

4. $\operatorname{tr}_{\mathcal{V} \otimes \mathcal{V}}\left(B_{i i} \otimes B_{j j} K\right)=0, \quad \forall i, j \in\{1,2, \ldots, N\}$.

This condition reflects the consistency conditions (2.1), i.e. $d_{X}\left(B_{i i}, B_{j j}\right)=0 \forall i \neq j$, 
and the required equality of the probability functions, i.e. $d_{X}\left(B_{i i}, B_{i i}\right)=p\left(B_{i i}\right)$.

To this end a calculation shows that

$$
B_{i i} \otimes B_{j j} K=\sum_{\nu, \rho=1}^{N} \delta_{\nu i} \delta_{j \nu} \kappa_{\nu \rho} B_{i \rho} \otimes B_{j \rho},
$$

where $\delta_{i j}$ is the Kronecker delta. But there is no way to get rid of the $\delta$ 's if $i \neq j$. This means in particular that this expression is the zero operator whose trace is trivially zero. In the case $i=j$, one shows that $\operatorname{tr}_{\mathcal{V} \otimes \mathcal{V}}\left(B_{i i} \otimes B_{i i} K\right)=\kappa_{i i}=0$.

Therefore the operators $X$ indeed define decoherence functionals via the rule $d_{X}(\alpha, \beta)=$ $\operatorname{tr}_{\mathcal{V} \otimes \mathcal{V}}(\alpha \otimes \beta X)$ with the required properties.

Consider now the case when $I_{n}$ is a partition of unity, which does not consist of onedimensional projectors. Then clearly the boolean subalgebra $\mathcal{A}_{I_{n}}$ with $n \leq N$ can be embedded into a boolean algebra $\mathcal{A}_{I_{N}}$ which is generated by one-dimensional projectors $I_{N}=\left\{\alpha_{1}, \alpha_{2}, \ldots, \alpha_{N}\right\}$. Therefore this Lemma suffices to prove the following proposition.

\section{Proposition}

Let the $\operatorname{dim} \mathcal{V}=N<\infty$. There exists a one-to-many map $\left(\mathcal{A}_{I_{n}}, \operatorname{Prob}\left(\mathcal{A}_{I_{n}}\right)\right) \mapsto d_{I_{N}}$ so that for any pair of a boolean subalgebra $\mathcal{A}_{I_{n}}$ of the space of history propositions $\mathcal{U} \mathcal{P}$ and a probability distribution on this subalgebra there exist decoherence functionals $d_{I_{N}} \in \mathcal{D}$, such that the pairs $\left(\mathcal{A}_{I_{n}}, \operatorname{Prob}\left(\mathcal{A}_{I_{n}}\right)\right)$ can be obtained from consistent sets with respect to those $d_{I_{N}} \in \mathcal{D}$ with $\mathcal{A}_{I_{n}} \subset \mathcal{A}_{I_{N}}$.

\section{DISCUSSION}

\section{A. The need for a more refined analysis of the properties of decoherence functionals}

The theorem established in the last section is mainly an existence proof. It does not determine all decoherence functionals fulfilling the requirements of yielding the given probability function $p(\alpha)$ on a given algebra $\mathcal{A}_{I_{N}}$. This is because, at present, no choice of consistency conditions is known which allows us to find all boolean algebras on which $p_{d}(\alpha)$ is a probability function. The explicit construction above of examples of such $d_{I_{N}} \in \mathcal{D}$, which have a remarkably simple form, says that this subset is already 'complete' in the sense that any probability distribution on the boolean algebra generated by any partition of the unity in the space of histories $\mathcal{U P}$ can be given by evaluating the probability function of certain decoherence functionals $d_{I_{N}}$ on this algebra.

The proposition also does not show that each decoherence functional posesses consistent sets. But it has some curious implications which, for reasons of notational simplicity, 
we will discuss only for the finite-dimensional case.

Take any decoherence functional $d \in \mathcal{D}$. Determine its collection of consistent sets $\left\{I_{n_{j}}^{d}\right\}, n \in\{1,2, \ldots, N\}$ and $j \in \mathbb{N}$, of which there are $j$ sets with $n$ elements, with the corresponding probability function $p_{d}(\alpha)$. Usually such a consistent set will not consist of $N$ one-dimensional projectors: it will rather be 'coarse grained' in the sense that the dimension of some of the projectors will be greater than one, for example for $d_{(H, \rho)}(\alpha, \beta)=\operatorname{tr}_{\mathcal{H}}\left(\tilde{C}_{\alpha}^{\dagger} \rho_{t_{0}} \tilde{C}_{\beta}\right)$. Embed each boolean algebra $\mathcal{A}_{I_{n_{j}}^{d}}$ generated by one of these coarse-grained consistent sets into an algebra $\mathcal{A}_{I_{N}}$ generated by a partition of unity into one-dimensional projectors $I_{N}$. This can always be done in many different ways. Then the theorem asserts that we can find at least one simple decoherence functional $d_{I_{N}}$ which gives the same probability function on the subalgebra of the boolean algebra generated by $I_{N}$ which is generated by the elements of $I_{n_{j}}^{d}$. Notice that if the consistent set is coarse grained, there exist uncountably many $d_{I_{N}}$ having this property.

Therefore we have shown that a single pair of a consistent set and a probability function is in general not sufficient to uniquely associate with it a decoherence functional. We therefore need additional criteria in order to establish such a correspondence.

Two options appear at present:

- The embeddings just mentioned exist for each single consistent set, but in general there will not be a single embedding for all the consistent sets belonging to one decoherence functional. One could therefore start with a collection of partitions of unity in $\mathcal{U P}$ and ask if there is a decoherence functional uniquely associated with it, such that these sets are consistent.

- Try to distinguish decoherence functionals by their 'symmetry groups'. This is a notion that will be developed elsewhere. It could subsume the work on symmetries, equivalence of consistent sets etc. [8, 9, 10, 11] to tackle the mentioned problem.

\section{B. Implications for the history version of standard quantum mechanics}

An example of an orthoalgebra $\mathcal{U P}$ is provided by standard quantum mechanics. In this case the set of all history-propositions $\mathcal{U P}$ can indeed be identified with the lattice of projectors on a certain Hilbert space or a subset thereof. Recall that one associates the homogeneous history $\alpha=\left(\alpha_{t_{1}}, \alpha_{t_{2}}, \ldots, \alpha_{t_{n}}\right)$ with a genuine projection operator $\alpha_{t_{1}} \otimes \alpha_{t_{2}} \otimes \cdots \otimes \alpha_{t_{n}}$ on the tensor product space $\mathcal{V}_{n}:=\mathcal{H}_{t_{1}} \otimes \mathcal{H}_{t_{2}} \otimes \cdots \otimes \mathcal{H}_{t_{n}}$ of $n$ copies of the Hilbert space $\mathcal{H}$ on which the canonical theory is defined. The final Hilbert space is obtained by forming the infinite-dimensional tensor product $\mathcal{V}:=\otimes_{t \in \mathbb{R}}^{\Omega} \mathcal{H}_{t}$ as indicated in $\llbracket$. Since this space is itself not separable one has to be cautious about drawing immediate conclusions from the result for this case. However, there is a sense in which it is valid. 
In [6] it had been shown that there exists an operator $X_{(H, \rho)}$ on $\mathcal{V} \otimes \mathcal{V}$ so that

$$
d_{(H, \rho)}(\alpha, \beta)=\operatorname{tr}_{\mathcal{H}}\left(\tilde{C}_{\alpha}^{\dagger} \rho_{t_{0}} \tilde{C}_{\beta}\right)=\operatorname{tr}_{\mathcal{V} \otimes \mathcal{V}}\left(\alpha \otimes \beta X_{(H, \rho)}\right) .
$$

In particular if we fix a temporal support $t_{1}<t_{2}<\cdots<t_{n}$ we can give the operator explicitly on $\mathcal{V}_{n} \otimes \mathcal{V}_{n}$, where $\mathcal{V}_{n}$ is the separable Hilbert space mentioned before. If therefore a consistent set of homogeneous history propositions can be found for this temporal support, one can equally well describe it by many other decoherence functionals on $\mathcal{V}_{n} \otimes \mathcal{V}_{n}$ so that $d(\alpha, \alpha)=d_{(H, \rho)}(\alpha, \alpha)$, albeit not ones which are of the standard form (4.1). Here we see once more the need to consider all decoherence functionals.

Let us use the example above to illustrate the kind of 'programme' to be followed in specifying a decoherence functional.

1. Firstly, we would like to find a set of (functional) equations $\left\{f^{i}(X)=0\right\}_{i=1}^{l}$ which determine $X$ to be of the form $X_{(H, \rho)}$.

2. Secondly, we then need to find properties of this $X_{(H, \rho)}$ which characterise the Hamiltonian $H$ and the initial density operator $\rho$. It is at this point one expects symmetry properties of $X$ to play a key role.

This procedure is conceptually very clear. One has to successively specify properties of decoherence functionals in addition to their defining ones. Once this task has been solved, one can ask for selection mechanisms among the consistent sets of history propositions belonging to a particular decoherence functional.

As indicated in the previous section one can certainly try to postulate the consistent sets of history propositions and/or the behavior of the probability function one would like a decoherence functional to possess in order to accomplish the task of specifying it uniquely by these means. Suggestions in this direction have been made by Isham and Linden in [5. Omnès mentions in his book [12] a result along similar lines of thought. But note that he is already assuming a 'unitary evolution' scenario, thereby excluding all the 'non-standard' decoherence functionals from the very start. But it is certainly of interest if his statement can, under appropriate assumptions, be translated into this new mathematical framework.

As a first step, as has been suggested by the referee, it would be interesting to see if for each partition of the unit operator into homogeneous history propositions, there exists always a standard decoherence functional, given by some $X_{(H, \rho)}$, such that this set is a consistent one with a given probability function. This is a topic for future research.

\section{CONCLUSION}

In assessing the value of the decoherent history programme with respect to its potentiality to resolve issues mentioned in the introduction it is undoubtably necessary to submit its 
two basic ingredients, the space of histories $\mathcal{U P}$ and the space of decoherence functionals $\mathcal{D}$, to a thorough investigation. The work on the properties of $\mathcal{U P}$ was started in references [4, 5]. A systematic approach to the properties of the space $\mathcal{D}$ had been initiated in [6] by the proof of a classification theorem for decoherence functionals under well defined circumstances.

The present article employs this result to show a completeness property of the space of decoherence functionals: there are 'enough' decoherence functionals to give each pair consisting of a partition of unity in $\mathcal{U P}$ and a probability function on the corresponding algebra the status of a consistent set.

Its importance lies in the fact that it calls for an additional concept, supplementary to the one of 'consistency' or 'decoherence', to establish a correspondence between physical systems and decoherence functionals which is not so general as to be void of any content. We need to know more about the properties of decoherence functionals.

It is likely that these new concepts involve notions like 'symmetry groups of decoherence functionals'. To be able to give a sensible meaning to those expressions one has to develop an analogue of Dirac's transformation theory for History Theories, a task currently under investigation.

\section{Acknowledgements}

It is a pleasure to thank Prof. C.J.Isham for useful discussions. I also acknowledge support through a DAAD fellowship HSPII financed by the German Federal Ministry of Research and Technology.

\section{References}

[1] R.B. Griffiths. Consistent histories and the interpretation of quantum mechanics. J. Stat. Phys., 36:219-272, 1984.

[2] R. Omnès. Logical reformulation of quantum mechanics. I. Foundations. J. Stat. Phys., 53:893-932, 1988.

[3] M. Gell-Mann and J. Hartle. Quantum mechanics in the light of quantum cosmology. In S. Kobayashi, H. Ezawa, Y. Murayama, and S. Nomura, editors, Proceedings of the Third International Symposium on the Foundations of Quantum Mechanics in the Light of New Technology, pages 321-343. Physical Society of Japan, Tokyo, 1990.

[4] C.J. Isham. Quantum logic and the histories approach to quantum theory. J. Math. Phys., 35: 2157-2185 (1994). 
[5] C.J. Isham and N. Linden. Quantum temporal logic and decoherence functionals in the histories approach to generalised quantum theory. J. Math. Phys., 35: 5452-5476 (1994).

[6] C.J. Isham, N. Linden and S. Schreckenberg. The classification of decoherence functionals: An analogue of Gleason's theorem. J. Math. Phys., 35: 6360-6370 (1994).

[7] R. Omnès. Consistent interpretations of quantum mechanics. Rev. Mod. Phys., 64:339382, 1992.

[8] M. Gell-Mann and J. Hartle. Equivalent sets of Histories and Multiple Quasiclassical Domains. gr-qc/9404013

[9] J. Hartle, R. Laflamme and D. Marolf. Conservation Laws in the Quantum Mechanics of Closed Systems. gr-qc/9410006

[10] F. Dowker and A. Kent. Properties of Consistent Sets. gr-qc/9409037

[11] F. Dowker and A. Kent. On the Consistent Histories Approach to Quantum Mechanics. gr-qc/9412067

[12] R. Omnès. The Interpretation of Quantum Mechanics. Princton University Press, 1994.

[13] M. Gell-Mann and J. Hartle. Classical equations for quantum systems. 1992. UCSB preprint UCSBTH-91-15.

[14] J. Hartle. Spacetime quantum mechanics and the quantum mechanics of spacetime. In Proceedings on the 1992 Les Houches School, Gravitation and Quantisation. 1993. 